\title{
SVD Aided Joint Transmitter and Receiver Design for the Uplink of Multiuser Detection Assisted MIMO Systems
}

\author{
W. Liu, L. L. Yang and L. Hanzo \\ School of ECS, University of Southampton, SO17 1BJ, United Kingdom. \\ Tel: +44-23-8059 6671, Fax: +44-23-8059 4508 \\ Email: $\{$ wl03r,lly,lh\}@ecs.soton.ac.uk, http://www-mobile.ecs.soton.ac.uk
}

\begin{abstract}
A novel singular value decomposition (SVD) aided uplink (UL) multiuser MIMO system is proposed. In contrast to the traditional minimum mean square error (MMSE) or zeroforcing (ZF) multiuser detection (MUD) technique, the proposed method exploits the specific characteristics of the individual users' channel matrix, instead of treating all the users' channels jointly. Furthermore, two different power allocation schemes are investigated in the context of the proposed structure. One of them was designed for achieving the maximum information rate, while the other for maintaining the maximum signal-to-noise ratio (SNR). We demonstrate that the capacity of the proposed scheme using the maximum information rate based power allocation policy is higher than that of the classic ZF receiver for the UL.
\end{abstract}

\section{INTRODUCTION}

In the uplink (UL) of MIMO-aided multiple-user systems both the multiple access interference (MAI) as well as interantenna interference (IAI) has to be mitigated. However, the employment of the optimum ML receiver may be excessively complex =even at the BS, where a higher complexity is tolerable. By contrast, the linear MMSE or ZF receiver [1] is unable to take the individual users' specific channel into account. As a further design alternative, in [2] an MMSE-based criterion was used for formulating both the preprocessing and postprocessing matrix, where close cooperation of the MSs was required. As another design alternative, in [3] the so-called maximum ratio [4] UL transmission scheme was investigated, where not all, but only the dominant right-hand-side (rhs) and left-hand-side (lhs) singular eigen vectors were adopted as the preprocessing and postprocessing eigen vectors, hence increasing the achievable diversity gain at the cost of reducing the multiplexing gain.

It has been shown in [5] that when accurate and prompt channel state information (CSI) is available at both the transmitter and receiver, singular value decomposition (SVD)-based adaptive modulation (AM) techniques applied in the context of MIMO systems are capable of achieving a high average spectral efficiency (ASE). Moreover, both SVD-assisted space time block coding (STBC) based transmit diversity schemes and V-BLAST-type spatial multiplexing arrangements have found numerous applications [6], [7]. However, these proposals were based on point-to-point communcations. In the context of multiple users, SVD based MUD was discussed in

The financial support of the European Union under the auspices of the Newcom and Phoenix projects, as well as that of the EPSRC UK is gratefully acknowledged.
[8], [9], when only the largest eigenvalue was invoked for UL transmission.

In this paper, SVD-based Space Division Multiple Access (SDMA) MUDs designed for UL reception are investigated. When using combined SVD-based preprocessing as well as postprocessing and assuming that the Channel Impulse Response (CIR) of all users is perfectly known by the MUD at the instant of transmission and reception, respectively, then the effect of both the MAI and IAI can be perfectly eliminated in the UL, since all signal links are uniquely and unambiguously identified by their CIRs. The proposed algorithm facilitates the employment of AM in the context of MIMO-aided multiple users and allows the extension of SVD-assisted STBC and VBLAST to multiple user scenarios. Furthermore, the proposed algorithm can be readily extendted to the case of downlink (DL) transmissions [10].

\section{SVD-BASED Uplink TRANSMISSION AND Detection}

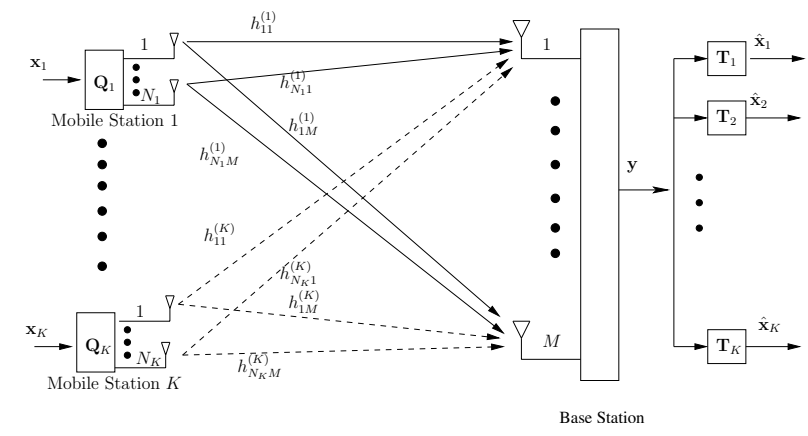

Fig. 1. Schematic of an UL multiuser MIMO system, where the BS employs $M$ receive antennas, while the MSs may employ different number of transmit antennas.

In this section we consider both UL transmission and detection in a multiuser MIMO system, where the base-station (BS) supports multiple mobile-stations (MSs). Although the extension of these principles to other types of MIMO systems is straightforward, the multiuser MIMO system considered here is in fact a SDMA system, where both the BS and MSs may employ multiple antennas both for reception and transmission. In our study we assume that the BS is capable of acquiring the UL CIRs of all the UL users. By contrast, a MS is only capable of acquiring an estimate of the UL CIR of 
itself for its own future instant of transmission. Furthermore, we assume that there is no cooperation among the UL users.

The schematic of the UL multiuser MIMO system considered in this paper is shown in Fig. 1, where the BS employs $M$ number of receive antennas and the $k$ th $(k=1,2, \ldots, K)$ MS uses $N_{k}$ number of transmit antennas. In Fig. $1 \boldsymbol{Q}_{k}$ $(k=1,2, \ldots, K)$ represents the UL MS transmitter's preprocessing matrix formulated for the transmission of the $k$ th MS's data $\boldsymbol{x}_{k}$. In Fig. $1 \boldsymbol{T}_{k}(k=1,2, \ldots, K)$ represents the receiver's post-processing matrix formulated for detecting the data transmitted by the $k$ th MS.

Let the $N_{k}$ data sysmbols to be transmitted by the $k$ th MS to the BS be hosted by a vector expressed as [2] $\boldsymbol{x}_{k}=\left[x_{k 1}, x_{k 2}, \cdots, x_{k N_{k}}\right]^{T}, k=1,2, \ldots, K$. As shown in Fig. $1, x_{k}$ is preprocessed using the $k$ th MS's transmitter preprocessing matrix $\boldsymbol{Q}_{k}$, yielding the output [2]

$$
\boldsymbol{d}_{k}=\boldsymbol{Q}_{k} \boldsymbol{x}_{k}, k=1,2, \ldots, K \text {. }
$$

Let the CIR matrix connecting the $N_{k}$ UL transmit antennas of the $k$ th MS with the $M$ UL receive antennas at the BS be expressed as

$$
\boldsymbol{H}_{k}=\left[\begin{array}{cccc}
h_{11}^{(k)} & h_{12}^{(k)} & \ldots & h_{1 N_{k}}^{(k)} \\
h_{21}^{(k)} & h_{22}^{(k)} & \ldots & h_{2 N_{k}}^{(k)} \\
\vdots & \vdots & \ddots & \vdots \\
h_{M 1}^{(k)} & h_{M 2}^{(k)} & \cdots & h_{M N_{k}}^{(k)}
\end{array}\right], k=1,2, \ldots, K,
$$

which is a $\left(M \times N_{k}\right)$-component matrix. Then, the received length- $M$ UL observation vector $\boldsymbol{y}$ at the $\mathrm{BS}$ can be expressed as [2]

$$
\boldsymbol{y}=\sum_{k=1}^{K} \boldsymbol{H}_{k} \boldsymbol{d}_{k}+\boldsymbol{n}=\sum_{k=1}^{K} \boldsymbol{H}_{k} \boldsymbol{Q}_{k} \boldsymbol{x}_{k}+\boldsymbol{n},
$$

where $\boldsymbol{n}$ is a length- $M$ noise observation vector, which is assumed to be Gaussian distributed with a zero mean and a covariance matrix given by $\sigma^{2} \boldsymbol{I}_{M}$.

Let us define

$$
\begin{aligned}
\boldsymbol{H} & =\left[\boldsymbol{H}_{1}, \boldsymbol{H}_{2}, \cdots, \boldsymbol{H}_{K}\right]^{T}, \\
\boldsymbol{Q} & =\operatorname{diag}\left\{\boldsymbol{Q}_{1}, \boldsymbol{Q}_{2}, \cdots, \boldsymbol{Q}_{K}\right\}, \\
\boldsymbol{d} & =\left[\boldsymbol{d}_{1}^{T}, \boldsymbol{d}_{2}^{T}, \cdots, \boldsymbol{d}_{K}^{T}\right]^{T}, \\
\boldsymbol{x} & =\left[\boldsymbol{x}_{1}^{T}, \boldsymbol{x}_{2}^{T}, \cdots, \boldsymbol{x}_{K}^{T}\right]^{T},
\end{aligned}
$$

where $H$ is the $\left(\sum_{k=1}^{K} N_{k} \times M\right)$-component combined channel matrix of the uplink, $Q$ is the $\left(\sum_{k=1}^{K} N_{k} \times \sum_{k \overline{\bar{K}}_{1}}^{K} N_{k}\right)$ component preprocessing matrix, $\boldsymbol{d}$ is the length- $\left(\sum_{k=1}^{\bar{K}} N_{k}\right)$ preprocessed data vector and $x$ is the length- $\left(\sum_{k=1}^{K} N_{k}\right)$ transmitted data vector. Then, (3) can also be written as

$$
y=H d+n=H Q x+n .
$$

As shown in Fig. 1, at the BS's UL receiver the $k$ th MS's transmitted UL data is recovered by processing the observation vector $\boldsymbol{y}$ using a $\left(N_{k} \times M\right)$-component weight matrix $\boldsymbol{T}_{k}$, which can be expressed as

$$
\hat{\boldsymbol{x}}_{k}=\boldsymbol{T}_{k} \boldsymbol{y}, k=1,2, \ldots, K .
$$

Let us collect all the data estimates of the $K$ UL users into a single vector $\hat{x}$ as

$$
\hat{\boldsymbol{x}}=\left[\hat{\boldsymbol{x}}_{1}^{T}, \hat{\boldsymbol{x}}_{2}^{T}, \cdots, \hat{\boldsymbol{x}}_{K}^{T}\right]^{T}=\boldsymbol{T} \boldsymbol{y}=\boldsymbol{T H} \boldsymbol{Q} \boldsymbol{x}+\boldsymbol{T n},
$$

where the overall $\left(\sum_{k=1}^{K} N_{k} \times M\right)$-component weight matrix is given by $\boldsymbol{T}=\left[\boldsymbol{T}_{1}^{T}, \boldsymbol{T}_{2}^{T}, \ldots, \boldsymbol{T}_{K}^{T}\right]^{T}$.

For the traditional zero-forcing receiver [1], which does not use transmit preprocessing, the overall preprocessing matrix $Q$ and the overall postprocessing matrix $T$ are expressed, respectively, as

$$
\begin{gathered}
\boldsymbol{Q}=\boldsymbol{I}_{\sum_{k=1}^{K} N_{k}}, \\
\boldsymbol{T}=\left(\boldsymbol{H}^{H} \boldsymbol{H}\right)^{-1} \boldsymbol{H}^{H} .
\end{gathered}
$$

Upon substituting (8) and (9) into (7), we have

$$
\hat{x}=x+n^{\prime},
$$

where

$$
\boldsymbol{n}^{\prime}=\left(\boldsymbol{H}^{H} \boldsymbol{H}\right)^{-1} \boldsymbol{H}^{H} \boldsymbol{n} .
$$

The covariance matrix $R$ of $\boldsymbol{n}^{\prime}$ is given by

$$
\boldsymbol{R}=E\left[\boldsymbol{n}^{\prime}\left(\boldsymbol{n}^{\prime}\right)^{H}\right]=\sigma^{2}\left(\boldsymbol{H}^{H} \boldsymbol{H}\right)^{-1} .
$$

Above we have derived the expression of the discrete-time signal received at the BS, as shown in (5), when the MS's UL transmitter employs transmitter preprocessing. Following the receiver post-processing stage of Fig. 1, the decision variable vector representing the transmitted data of the $K$ uplink users is given by (7). Below we consider both UL preprocessing as well as detection and derive the corresponding expressions for both the transmitter preprocessing matrix $\boldsymbol{Q}_{k}$ and for the receiver post-processing matrix $T_{k}$ using the classic SVD principles. In our derivation we assume that we have $M \geq \sum_{k=1}^{K} N_{k}$, which physically means that the number of antennas at the BS is equal to or higher than the sum of antennas employed by all the $K$ MSs. Let us assume that $\boldsymbol{H}_{k}$ of (2) satisfies $\operatorname{rank}\left(\boldsymbol{H}_{k}\right)=N_{k}$. Then, the SVD of $\boldsymbol{H}_{k}$ can be expressed as

$\boldsymbol{H}_{k}=\boldsymbol{U}_{k}\left[\begin{array}{c}\boldsymbol{\Lambda}_{k}^{1 / 2} \\ \mathbf{0}\end{array}\right] \boldsymbol{V}_{k}^{H}=\left[\begin{array}{ll}\boldsymbol{U}_{k s} & \boldsymbol{U}_{k n}\end{array}\right]\left[\begin{array}{c}\boldsymbol{\Lambda}_{k}^{1 / 2} \\ \mathbf{0}\end{array}\right] \boldsymbol{V}_{k}^{H}=\boldsymbol{U}_{k s} \boldsymbol{\Lambda}_{k}^{1 / 2} \boldsymbol{V}_{k}^{H}$,

where $\boldsymbol{U}_{k}$ and $\boldsymbol{V}_{k}$ are $(M \times M)$ and $\left(N_{k} \times N_{k}\right)$ component unitary matrices, respectively, while $\Lambda=$ $\operatorname{diag}\left\{\lambda_{1}, \lambda_{2}, \cdots, \lambda_{N_{k}}\right\}$ contains the $N_{k}$ non-zero eigenvalues of $\boldsymbol{H}_{k}^{H} \boldsymbol{H}_{k}$ or $\boldsymbol{H}_{k} \boldsymbol{H}_{k}^{H}$. Furthermore, in (13) the columns of $\boldsymbol{U}_{k}$ are constituted by the eigenvectors of $\boldsymbol{H}_{k} \boldsymbol{H}_{k}^{H}, \boldsymbol{U}_{k s}$ consists of the $N_{k}$ eigenvectors corresponding to the signal subspace of $\boldsymbol{H}_{k} \boldsymbol{H}_{k}^{H}$, while $\boldsymbol{U}_{k n}$ consists of the $\left(M-N_{k}\right)$ eigenvectors corresponding to the null subspace of $\boldsymbol{H}_{k} \boldsymbol{H}_{k}^{H}$. Similarly, the columns of $\boldsymbol{V}_{k}$ correspond to the eigenvectors of $\boldsymbol{H}_{k}^{H} \boldsymbol{H}_{k}$.

Upon substituting (13) into (3), the vector $y$ of the UL received signal of Figure 1 can be expressed as

$$
\boldsymbol{y}=\sum_{k=1}^{K} \boldsymbol{U}_{k s} \boldsymbol{\Lambda}_{k}^{1 / 2} \boldsymbol{V}_{k}^{H} \boldsymbol{Q}_{k} \boldsymbol{x}_{k}+\boldsymbol{n}
$$


where the channel matrix $\boldsymbol{H}_{k}$ of the $k$ th user is replaced by its SVD. Let the transmitter preprocessing matrix $\boldsymbol{Q}_{k}$ of Figure 1 be formulated as

$$
\boldsymbol{Q}_{k}=\boldsymbol{V}_{k} \boldsymbol{\beta}_{k}, k=1,2, \ldots, K,
$$

where $\boldsymbol{\beta}_{k}=\operatorname{diag}\left\{\beta_{k 1}, \cdots, \beta_{k N_{k}}\right\}$ is a $\left(N_{k} \times N_{k}\right)$-component diagonal matrix, which is employed for implementing the transmission power-allocation, as we will discuss in detail in Subsection III. After substituting (15) into (14) and exploiting the property $\boldsymbol{V}_{k}^{H} \boldsymbol{V}_{k}=\boldsymbol{I}_{N_{k}}$, the vector $\boldsymbol{y}$ of the UL received signal seen in Figure 1 can be simplified to

$$
\boldsymbol{y}=\sum_{k=1}^{K} \boldsymbol{U}_{k s} \boldsymbol{\Lambda}_{k}^{1 / 2} \boldsymbol{\beta}_{k} \boldsymbol{x}_{k}+\boldsymbol{n}
$$

where the rhs singular vectors of the channel matrix $\boldsymbol{H}_{k}$ of the $k$ th UL transmitter has been cancelled out by the corresponding UL preprocessing matrix $\boldsymbol{Q}_{k}$ of Figure 1 at the $k$ th UL MS transmitter.

Equation (16) shows that the UL transmit preprocessing matrix $\boldsymbol{Q}_{k}$ of (15) decouples each of the antenna-specific transmitted data symbols of the $k$ th $\mathrm{MS}$, from those of its other antennas.

Let us define

$$
\begin{aligned}
\boldsymbol{U}_{s} & =\left[\boldsymbol{U}_{1 s}, \boldsymbol{U}_{2 s}, \cdots, \boldsymbol{U}_{K s}\right], \\
\boldsymbol{\Lambda}^{1 / 2} & =\operatorname{diag}\left\{\boldsymbol{\Lambda}_{1}^{1 / 2}, \boldsymbol{\Lambda}_{2}^{1 / 2}, \cdots, \boldsymbol{\Lambda}_{K}^{1 / 2}\right\}, \\
\boldsymbol{\beta} & =\operatorname{diag}\left\{\boldsymbol{\beta}_{1}, \boldsymbol{\beta}_{2}, \cdots, \boldsymbol{\beta}_{K}\right\} .
\end{aligned}
$$

Then, the received UL signal vector $\boldsymbol{y}$ of Figure 1 can be expressed as

$$
\boldsymbol{y}=\boldsymbol{U}_{s} \Lambda^{1 / 2} \boldsymbol{\beta} \boldsymbol{x}+\boldsymbol{n} .
$$

Note that although the columns of $\boldsymbol{U}_{k s}(k=1,2, \ldots, K)$ are orthogonal, suggesting that there is no IAI, the columns of $U_{s}$ in (18) corresponding to the different UL MS transmitters are non-orthogonal. Therefore, there is MAI, which should be cancelled by the BS's receiver.

Upon substituting (18) into (7), we arrive at

$$
\hat{x}=T U_{s} \Lambda^{1 / 2} \beta x+T n .
$$

It can be shown that there are many alternatives for the design of the BS's UL receiver post-processing matrix $T$, as discussed in [1]. As an example, in this paper we focus our attention on the ZF UL MUD scheme, which is a linear detector and is capable of entirely eliminating the MAI, although at the cost of potential noise-enhancement.

The ZF UL MUD solution encapsulated in $T$ can be readily derived in the context of [1]

$$
\boldsymbol{T}=\left[\boldsymbol{U}_{s}\right]^{+}=\left(\boldsymbol{U}_{s}^{H} \boldsymbol{U}_{s}\right)^{-1} \boldsymbol{U}_{s}^{H},
$$

where $[\cdot]^{+}$denotes the pseudo inverse of the matrix $U_{s}$. Upon substituting (20) into (19), we arrive at

$$
\hat{x}=\Lambda^{1 / 2} \beta x+n^{\prime} .
$$

Explicitly, the MAI is entirely removed. In (21) the noise term $n^{\prime}=T n$, which still represents a Gaussian noise vector with zero mean, but its covariance matrix is given by

$$
E\left[\boldsymbol{n}^{\prime}\left(\boldsymbol{n}^{\prime}\right)^{H}\right]=\sigma^{2}\left(\boldsymbol{U}_{s}^{H} \boldsymbol{U}_{s}\right)^{-1}
$$

which indicates that the noise observations become correlated after the ZF MUD receiver post-processing.

Since the antenna-specific signals transmitted from a given MS are decoupled by its transmitter preprocessing, it can be readily shown that the diagonal entries of $\boldsymbol{U}_{s}^{H} \boldsymbol{U}_{s}$ are constituted by $K$ unity matrices having the sizes of $\left(N_{k} \times N_{k}\right)$ for $k=1,2, \ldots, K$, respectively. Therefore, a given MS does not impose correlation on its own antenna-specific noise samples. This property makes it possible for us to study the power-allocation for a specific MS without considering the correlation among the noise's observation samples.

\section{Power-AllocAtion}

In the context of UL transmission, the originally allocated transmission power of the $k$ th $\mathrm{MS}$ is given by $E\left[\left\|x_{k}\right\|^{2}\right]=$ $N_{k}$, where we assumed $E\left[\left\|x_{k i}\right\|^{2}\right]=1$. Hence the powerallocation is carried out under the constraint of

$$
E\left[\left\|\boldsymbol{\beta}_{k} \boldsymbol{x}_{k}\right\|^{2}\right] \leq E\left[\left\|\boldsymbol{x}_{k}\right\|^{2}\right]=N_{k},
$$

which means that the total transmission power after UL preprocessing cannot exceed the originally allocated power. In this section power-allocation is carried out for the UL multiuser MIMO system either based on maximizing the information rate of the individual MSs [11] or on maximizing the overall SNR [12].

1) Maximum Information Rate Based Power-Allocation: Since in the considered system there is no cooperation among the MSs, a MS can only exploit the knowledge of it own estimated UL CIR and its power can only be shared across to its own transmit antennas, while ignoring the existence of all the other MSs. In this case the 'water-filling' principle [11] may be employed for allocating the MS's total transmission power, in order to maximize its achievable information rate. Specifically, as shown in (21), the decision variable vector of the $k$ th MS can be expressed as

$$
\hat{\boldsymbol{x}}_{k}=\boldsymbol{\Lambda}_{k}^{1 / 2} \boldsymbol{\beta}_{k} \boldsymbol{x}_{k}+\boldsymbol{n}_{k}^{\prime}, k=1,2, \ldots, K .
$$

When treating the components of $\boldsymbol{n}_{k}^{\prime}$ as independent Gaussian random variables, it can be shown that, in order to maximize the achievable information rate, the power-allocation related matrix $\boldsymbol{\beta}_{k}$ should be chosen according to [11] as

$$
\beta_{k i}^{2}=\left(v_{k}-\frac{\sigma^{2}}{\lambda_{k i}}\right)^{+}, i=1,2, \ldots, N_{k},
$$

where $(x)^{+}$is defined as

$$
(x)^{+}= \begin{cases}x & \text { if } x \geq 0 \\ 0 & \text { if } x<0\end{cases}
$$

and $v_{k}$ is chosen so that (23) is satisfied. 
Upon exploiting the 'water-filling' principle [11], the maximum information rate normalized by the number of transmit antennas of MS $k$ can be expressed as [13]

$$
\mathcal{I}_{\max }\left(\hat{\boldsymbol{x}}_{k}, \boldsymbol{x}_{k}\right)=\frac{1}{N_{k}} \sum_{i=1}^{N_{k}} \log _{2}\left[\left(1+\frac{\lambda_{k i}}{\sigma^{2}}\left(v_{k}-\frac{\sigma^{2}}{\lambda_{k i}}\right)^{+}\right)\right] \text {, }
$$

which denotes the maximum number of error free information bits per channel use for the channel $\boldsymbol{H}_{k}$ at a given SNR, provided that the noise samples observed at the BS's receiver are independent.

When the power-allocation regime of (25) is invoked and assuming that the noise samples are correlated, the maximum achievable rate must be modified as follows. Let us denote the covariance matrix of the noise by

$$
\boldsymbol{R}_{k}=E\left[\boldsymbol{n}_{k}^{\prime}\left(\boldsymbol{n}_{k}^{\prime}\right)^{H}\right] .
$$

The maximum achievable normalized rate can be expressed as [14]

$$
\mathcal{I}_{\max }\left(\hat{\boldsymbol{x}}_{k}, \boldsymbol{x}_{k}\right)=\frac{1}{N_{k}} \log _{2}\left[\operatorname{det}\left(\boldsymbol{I}_{N_{k}}+\boldsymbol{\Lambda}_{k} \boldsymbol{\beta}_{k}^{2} \boldsymbol{R}_{k}^{-1}\right)\right] .
$$

From (29) we can see that the channel capacity associated with $\boldsymbol{H}_{k}$ is not treated as the sum of $N_{k}$ single input single output (SISO) channel's capacity, since the correlation among the noise components is known at the receiver and hence the receiver is capable of exploiting this correlation for improving the channel capacity of $\boldsymbol{H}_{k}$. The maximum attainable information rate of (29) is achieved, when the receiver is capable of exploiting the knowledge of $\boldsymbol{R}_{k}$ for the detection of the $k$ th user, despite having no knowledge concerning the correlation of the noise experienced by the different users, since there is no cooperation among the MSs.

Note furthermore that when the conventional zero-forcing detection of (10) is considered, the achievable normalized capacity can be expressed as [15]

$$
\mathcal{I}_{\max }(\hat{\boldsymbol{x}}, \boldsymbol{x})=\frac{1}{\sum_{k=1}^{K} N_{k}} \sum_{i=1}^{\sum_{k=1}^{K} N_{k}} \log _{2}\left[\left(1+\frac{1}{[\boldsymbol{R}]_{(i, i)}}\right)\right],
$$

where $R$ is the auto-correlation matrix of the noise given by (12).

2) Power-Allocation Designed for Achieving the Maximum SNR: For this scenario, we assume that the entries of the vector $\boldsymbol{n}_{k}^{\prime}$ are independent identically distributed (i.i.d) Gaussian random variables having a common variance of $\sigma^{2} / 2$ per dimension. Given the decision variable vector of (24), the SNR of the $i$ th antenna's symbol of MS $k$ can be expressed as

$$
\gamma_{k i}=\frac{\lambda_{k i} \beta_{k i}^{2}}{\sigma^{2}}, i=1,2, \ldots, N_{k} ; k=1,2, \ldots, K
$$

and the sum of the SNRs of all the $N_{k}$ antennas is given by $\gamma_{k}=\sum_{i=1}^{N_{k}} \gamma_{k i}$. However, it can be shown that maximizing $\gamma_{k}$ does not result in a meaningful solution [12]. Hence we opt for minimizing the following expression [12]

$$
\xi_{k}=\sum_{i=1}^{N_{k}} \frac{1}{\gamma_{k i}}=\sum_{i=1}^{N_{k}} \frac{\sigma^{2}}{\lambda_{k i} \beta_{k i}^{2}}
$$

\begin{tabular}{|l||l||l||l||l|}
\hline & Scheme 1 & Scheme 2 & Scheme 3 & Scheme 4 \\
\hline Number of MSs $(K)$ & 10 & 5 & 4 & 2 \\
\hline Number of antennas at each MS $\left(N_{k}\right)$ & 2 & 4 & 5 & 10 \\
\hline Number of antennas at the BS $(M)$ & 20 & 20 & 20 & 20 \\
\hline Modulation scheme & BPSK & BPSK & BPSK & BPSK \\
\hline
\end{tabular}

TABLE I

PARAMETERS FOR SDMA TRANSMISSION BASED ON SVD FOR VARIOUS FULLY LOADED SYSTEMS, WHERE THE MSS BENEFIT FROM HAVING $2,4,5$ OR 10 ANTENNAS.

under the constraint of obeying the total transmission power of (23)

After further mathematicl manipulations [12], we arrive at the power-allocation scheme optimized for maintaining the maximum achievable SNR in the form of

$$
\beta_{k i}^{2}=N_{k}\left(\sum_{i=1}^{N_{k}} \frac{1}{\sqrt{\lambda_{k i}}}\right)^{-1} \frac{1}{\sqrt{\lambda_{k i}}}, i=1,2, \ldots, N_{k} .
$$

\section{Performance Results}

In this section, simulation results are provided for characterizing the achievable performance of the proposed algorithm in conjunction with a specific power allocation scheme. Speficically, Table I outlines the parameters used for the various fully loaded systems ${ }^{1}$, where the MSs benefit from having $2,4,5$ or 10 antennas.

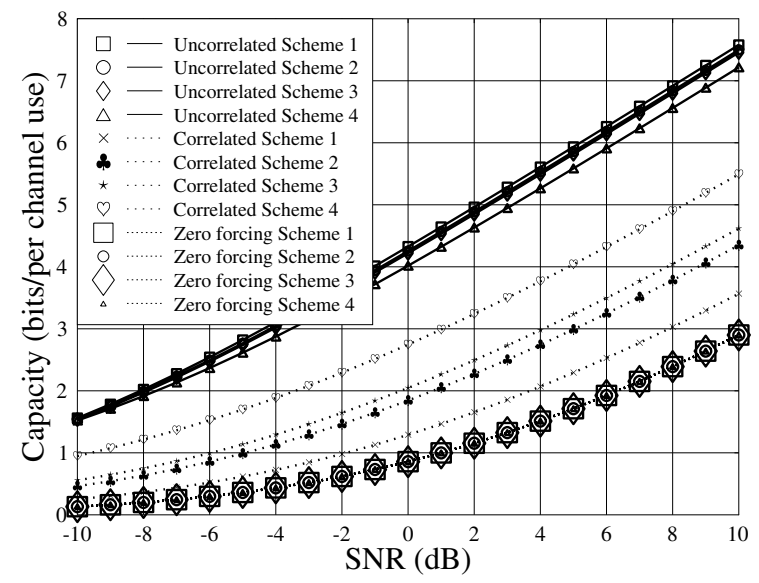

Fig. 2. The average capacity versus average SNR per symbol evaluted from (27) and (29) for UL transmission, when invoking the maximum information rate based power allocation policy for the four diffent transmission schemes outlined in Table I.

In Figure 2, the attainable average capacity versus average SNR per symbol recorded for UL transmission is plotted, when invoking the maximum information rate based power allocation policy of [11], [14] for the four diffent transmission schemes, of Table I. We can see from Figure 2 that for a specific scheme of Table I, the capacity achieved by assuming

\footnotetext{
${ }^{1}$ By fully loaded system, we mean the number of antennas at BS is equal
} to the sum of antennas of all MSs 
the presence of the uncorrelated noise of (27) is significantly higher than that associated with encountering the correlated noise of (29). This is due to the noise enhancement imposed by the postprocessing. Furthermore, the highest capacity is achieved by Schemes 1, 2, 3 and 4, when the presence of uncorrelated noise is assumed. This can be explained by the fact that the system capacity does not increase proportionally with the number of transmitter antennas. Moreover, when considering the correlated noise scenario of Figure 2, the more users the system supports, the lower the capacity it achieves. This is because for a fully loaded system a higher number of users results in lower number of antennas at each MS, resulting in a higher MUI for each individual user. Furthermore, the classic ZF receiver treats all other $\left(\sum_{k=1}^{K} N_{k}-1\right)$ transmit antennas' signal as interference, which results in the lowest performance.

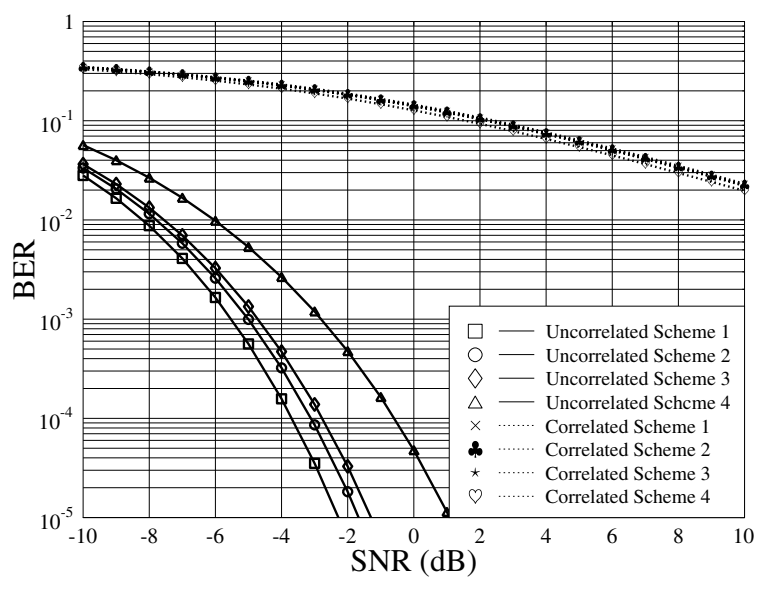

Fig. 3. Average BER versus average SNR per symbol for UL transmission, when invoking the maximum SNR based power allocation policy for the four different schemes of Table I.

In Figure 3 the achievable average BER versus average SNR per symbol performance is plotted for UL transmission, when invoking the maximum SNR based power allocation policy for the four different schemes of Table I. We can see from Figure 3 that the BER performance recorded for uncorrelated noise is always better than that corresponding to the correlated noise scenario, which again is due to the more substantial noise enhancement experienced in the presence of correlated noise. Furthermore, the BER performance degrades upon increasing the number of transmit antennas at the MS, when uncorrelated noise is encountered. The reason for this is because having more transmit antennas at the MS may result in a wider eigenvalue dynamic range. Consequently, low eigenvalues may be obtained, which dominate the BER performance. However, in the presence of correlated noise the BER performance is similar for the schemes considered in Table I. The reason for this is that when we have less antennas at the MS, we support more users, which results in more serious MUI.

\section{Summary AND CONCLUSIONS}

In this paper, SVD based SDMA MUD algorithms were proposed for UL reception. Based on the proposed algorithm, both the maximum information rate and the maximum SNR based power allocation policies of [11], [12], [14] were considered for UL transmisson and both the attainable capacity and the achievable BER performance was compared for both the uncorrelated and correlated noise scenarios. The simulation results of Figures 2 and 3 suggest that a performance loss is experienced due to the noise enhancement experienced in the correlated noise scenarios.

\section{REFERENCES}

[1] S. Verdu, Multiuser Detection. Cambridge University Press, 1998.

[2] S. Serberli and A. Yener, "Transceiver optimization for multiuser MIMO systems," IEEE Transactions on Signal Processing, vol. 52, pp. 214 226, January 2004.

[3] Y. Tokgoz and B. D. Rao, "Performance analysis of maximum ratio transmission based multi-cellular MIMO systems," IEEE Transactions on Wireless Communications, vol. 5, pp. 83 - 89, January 2006.

[4] T. K. Y. Lo, "Maximum ratio transmission," IEEE Transactions on Communications, vol. 47, pp. 1458 - 1461, October 1999.

[5] Z. Zhou and B. Vucetic, "MIMO systems with adaptive modulation," IEEE Transactions on Vehicular Technology, vol. 54, pp. 1828 - 1842, September 2005.

[6] S. X. Ng, B. L. Yeap, and L. Hanzo, "Full-rate, full-diversity adaptive space time block coding for transmission over Rayleigh fading channels," in IEEE Vehicular Technology Conference, (Stockholm, Sweden), pp. 1210 - 1214, 30 May - 1 June 2005.

[7] T. J. Willink, "An adaptive algorithm for V-BLAST," in IEEE Vehicular Technology Conference, (Stockholm, Sweden), pp. 2044 - 2048, 26 29 September 2004

[8] J. Kim and J. M. Cioffi, "Spatial multiuser access with antenna diversity using singular value decomposition," in IEEE International Conference on Communications, (New Orleans, LA, USA), pp. 1253 - 1257, 18-22 June 2000.

[9] K. Kim, S. Lee, and K. Chang, "An efficient multiuser access scheme combining the transmit diversity with the modified SVD methods for MIMO channels," in Conference Record of the Thirty-Sixth Asilomar Conference on Signals, Systems and Computers, (Pacific Grove, California, USA), pp. 1719 - 1721, 3 - 6 November 2002.

[10] W. Liu, L. L. Yang, and L. Hanzo, "SVD sssisted joint transmitter and receiver design for broadcasting MIMO systems," submitted to Proceedings of the IEEE Vehicular Technology Conference 2007 Spring, (Singapore), 11 - 14 May 2008.

[11] I. E. Telatar, "Capacity of multi-antenna Gaussian channels," European Transactions on Telecommunications, vol. 10, pp. 585-595, May 1999.

[12] M. Morelli and L. Sanguinetti, "A novel prefiltering technique for downlink transmissions in TDD MC-CDMA systems," IEEE Transactions on Wireless Communications, vol. 4, pp. 2064 - 2069, September 2005.

[13] B. Vucetic and J. Yuan, Space-Time Coding. Wiley, 2003.

[14] B. Holter, "On the capacity of the MIMO channel: A tutorial introduction," in IEEE Norwegian Symposium on Signal Processing, (Trondheim, Norway), pp. 167 - 172, 18 - 20 October 2001.

[15] R. W. Heath, M. Airy, and A. J. Paulraj, "Multiuser diversity for mimo wireless systems with linear receivers," in Conference Record of the Thirty-Fifth Asilomar Conference on Signals, Systems and Computers, (Pacific Grove, CA, USA), pp. 1194 - 1199, 4 - 7 Nov 2001. 